\title{
Scalar heat kernel with boundary in the worldline formalism
}

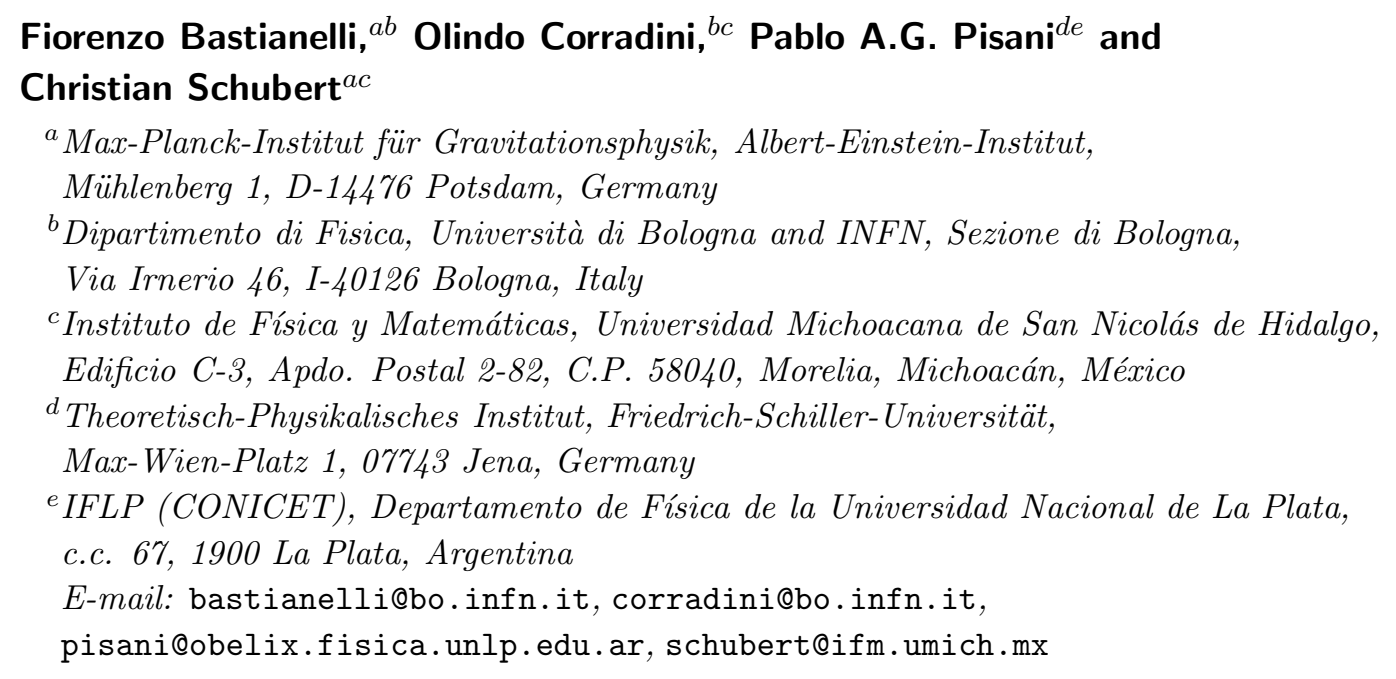

ABSTRACT: The worldline formalism has in recent years emerged as a powerful tool for the computation of effective actions and heat kernels. However, implementing nontrivial boundary conditions in this formalism has turned out to be a difficult problem. Recently, such a generalization was developed for the case of a scalar field on the half-space $\mathbb{R}_{+} \times$ $\mathbb{R}^{D-1}$, based on an extension of the associated worldline path integral to the full $\mathbb{R}^{D}$ using image charges. We present here an improved version of this formalism which allows us to write down non-recursive master formulas for the $n$-point contribution to the heat kernel trace of a scalar field on the half-space with Dirichlet or Neumann boundary conditions. These master formulas are suitable to computerization. We demonstrate the efficiency of the formalism by a calculation of two new heat-kernel coefficients for the half-space, $a_{4}$ and $a_{9 / 2}$.

Keywords: Field Theories in Lower Dimensions, Field Theories in Higher Dimensions, Sigma Models. 


\section{Contents}

1. Introduction 1

2. Heat kernel on the half-line: indirect contribution 5

2.1 The one-point function 6

2.2 The two-point function

3. Heat kernel on the half-line: direct contribution 9

3.1 The one-point function 10

3.2 The two-point function 11

4. Conclusions 14

A. Half-line heat kernel coefficients $a_{4}$ and $a_{9 / 2}$

B. Heat kernel on the half-space 16

\section{Introduction}

The "string-inspired" worldline formalism, originally developed in the context of QCD scattering amplitudes [1, 2], has during the last fifteen years evolved also into a powerful tool for the calculation of effective actions and heat kernels. One may recall that, generally, one-loop effective actions can be written in terms of determinants of certain differentials operators depending on given background fields. For the simplest and prototypical case, a (real) scalar field with self-interaction $\mathrm{U}(\phi)$, this operator is ${ }^{1}$

$$
H=-\square+U^{\prime \prime}(\phi)
$$

and the one-loop effective action can be written as

$$
\begin{aligned}
\Gamma[\Phi] & =\frac{1}{2} \ln \operatorname{Det} H=-\frac{1}{2} \int_{0}^{\infty} \frac{d T}{T} \operatorname{Tr} \mathrm{e}^{-T H} \\
& =-\frac{1}{2} \int_{0}^{\infty} \frac{d T}{T} \int d^{D} x K(T ; x, x)
\end{aligned}
$$

where $K(T ; x, x)$ is the diagonal of the heat kernel of the operator $H$,

$$
K(T ; x, y)=\left\langle x\left|\mathrm{e}^{-T H}\right| y\right\rangle
$$

\footnotetext{
${ }^{1}$ We use euclidean conventions throughout this paper.
} 
A standard way of calculating the effective action is given by the (diagonal) heat kernel expansion,

$$
K(T ; x, x)=(4 \pi T)^{-\frac{D}{2}} \sum_{n=0}^{\infty} a_{n}(x, x) T^{n}
$$

See [3 -5] for reviews on the results which have been obtained for the heat kernel coefficients $a_{n}$ in various field theories, as well as for their applications in quantum field theory.

In the worldline formalism, the starting point is the following worldline path integral representation of the effective action (1.2) (see, e.g., [6])

$$
\Gamma[\phi]=-\frac{1}{2} \int_{0}^{\infty} \frac{d T}{T} \int \mathcal{D} x \exp \left[-\int_{0}^{T} d \tau\left(\frac{1}{4} \dot{x}^{2}+V(x(\tau))\right)\right]
$$

where we now denote $U^{\prime \prime}(\phi(x))=: V(x)$. Here the path integral in (1.5) is over the space of all closed loops $x(\tau)$ in spacetime with periodicity $x(T)=x(0)$. It will be convenient to rescale the worldline action to the circle of unit length,

$$
\int_{0}^{T} d \tau\left(\frac{1}{4} \dot{x}^{2}+V(x(\tau))\right)=\frac{1}{4 T} \int_{0}^{1} d \tau \dot{x}^{2}+T \int_{0}^{1} d \tau V(x(\tau))
$$

In the application of (1.5) to the calculation of the effective action, the most straightforward approach is to expand the interaction exponential, Taylor expand $V$, and evaluate the resulting Gaussian integrals using a worldline Green's function adapted to the periodic boundary conditions. Since for periodic boundary conditions the path integral has perturbatively a zero mode, before doing so one has to split

$$
\begin{aligned}
x(\tau) & =x+y(\tau) \\
\int \mathcal{D} x & =\int d^{D} x \int \mathcal{D} y
\end{aligned}
$$

This leaves a dependence of the worldline Green's function on the boundary conditions chosen for $y(\tau)$. The main two choices are

1. (Worldline) Dirichlet boundary conditions (DBC), $y(0)=y(1)=0$. This leads to a worldline correlator $\left\langle y^{\mu}(\tau) y^{\nu}(\sigma)\right\rangle=-T \delta^{\mu \nu} G_{D}(\tau, \sigma)$, where

$$
\begin{aligned}
G_{D}(\tau, \sigma) & =2 \tau(\sigma-1) \theta(\sigma-\tau)+2 \sigma(\tau-1) \theta(\tau-\sigma) \\
& =|\tau-\sigma|+\frac{1}{2}(1-2 \tau)(1-2 \sigma)-\frac{1}{2}
\end{aligned}
$$

2. "String-inspired" boundary conditions $(\mathrm{SI}), \int_{0}^{1} d \tau y(\tau)=0$. This yields a correlator $\left\langle y^{\mu}(\tau) y^{\nu}(\sigma)\right\rangle=-T \delta^{\mu \nu} G_{S}(\tau, \sigma)$ with

$$
G_{S}(\tau, \sigma)=|\tau-\sigma|-(\tau-\sigma)^{2}
$$

where the coincidence limit has been subtracted; see discussion in [7, 6]. 
After this zero mode fixing, it is natural to Taylor expand $V$ at the point $x$,

$$
V(x+y)=\mathrm{e}^{y \cdot \partial} V(x)
$$

Combining $(1.10)$ with the expansion of the interaction exponential renders the path integral Gaussian. Formal Gaussian integration using either of the worldline correlators (1.8) or (1.9) then leads to the following master formula for the effective action,

$$
\Gamma[\Phi]=-\frac{1}{2} \int_{0}^{\infty} \frac{d T}{T} \int d^{D} x K_{D, S}(T, x)
$$

where

$$
\begin{aligned}
K_{D, S}(T, x)= & (4 \pi T)^{-\frac{D}{2}} \sum_{n=0}^{\infty} \frac{(-T)^{n}}{n !} \int_{0}^{1} d \tau_{1} \cdots \int_{0}^{1} d \tau_{n} \\
& \times \exp \left[-\frac{T}{2} \sum_{i, j=1}^{n} G_{D, S}\left(\tau_{i}, \tau_{j}\right) \partial_{i} \cdot \partial_{j}\right] V^{(1)}(x) \cdots V^{(n)}(x)
\end{aligned}
$$

Here it is understood that the derivative $\partial_{i}$ acts on $V^{(i)}$. The prefactor $(4 \pi T)^{-\frac{D}{2}}$ represents the free path integral determinant. As discussed in [7] (see also [6]), using the Dirichlet Green's function (1.8) in (1.11) reproduces precisely the heat kernel diagonal,

$$
K_{D}(T, x)=K(T ; x, x)
$$

On the other hand, $K_{S}(T, x)$ differs from $K(T ; x, x)$ by terms which are total derivatives. As it turns out, these total derivative terms have a simplifying effect in the sense that they lead to a more compact form of the effective action at higher orders of the heat kernel expansion [8, 9]. The master formula (1.11) with the string-inspired Green's function has been used in [9] for a calculation of $\Gamma[\Phi]$ to order $O\left(T^{8}\right)$. Both approaches have been extended to the effective action for quantum electrodynamics [10], nonabelian gauge theory [7, 11] and gravity 12, 13]. It must be mentioned, though, that the issue of the zero mode fixing becomes a much more nontrivial one in the curved space case 14.

All the work cited above pertains to the standard heat kernel, derived from operators defined on $\mathbb{R}^{D}$ or on a manifold without boundary. However, many important physics applications of effective actions involve nontrivial boundary conditions. The prime example is Casimir energies, for whose calculation there is presently still no sufficiently general method available in standard QFT, while there is increasing motivation not only from QED (see 15] for a review) but also from the physics of branes and field theories with extra dimensions. In particular, for a non supersymmetric brane configuration the Casimir force is an important ingredient for the analysis of the stability of the configuration [16]. During the last few years a variant of the worldline formalism based on a direct numerical calculation of the path integral 17] has been applied very successfully to the calculation of Casimir energies with Dirichlet boundary conditions [18]. This strongly suggests that one should study how to implement boundary conditions also in analytic versions of this formalism. Similarly, an important ingredient in the discovery of Hořava-Witten duality [19], a cornerstone of 
string theory, was the cancellation of Einstein anomalies in the ten-dimensional boundary of $\mathbb{R}^{10} \times \mathbf{S}^{1} / \mathbb{Z}_{2}$. Worldline models have so far been quite successfully used in the computation of anomalies in boundaryless manifolds [20, 12], so that a generalization to spaces with boundary seems in order.

For manifolds with a boundary, the expansion formula for the heat kernel trace

$$
\operatorname{Tr} \mathrm{e}^{-T H}=(4 \pi T)^{-\frac{D}{2}} \sum_{n} a_{n} T^{n}
$$

remains valid but now the integrated coefficients $a_{n}$ include boundary contributions, and also half-integer values of $n$ appear [21]. Various methods for computing the heat kernel trace on manifolds with boundary can be found in the literature [21- 27]; see also [4, 5] for reviews oriented towards physical applications.

As a first step in the direction of a generalization of worldline techniques to spaces with boundary, in 28] three of the present authors considered the heat kernel for a scalar field propagating on the half-space $\mathbb{R}_{+} \times \mathbb{R}^{D-1}$ and used the image charge method to write the heat kernel trace on the half space as a combination of heat kernels on the whole space. For the simple half-line $\mathbb{R}_{+}$(that already captures the main features of the method) with Dirichlet/Neumann boundary conditions this yields the following combination of whole line heat kernels

$$
\operatorname{Tr} \mathrm{e}^{-T H}=\int_{0}^{\infty} d x\left\langle x\left|\mathrm{e}^{-T H}\right| x\right\rangle \mp \int_{0}^{\infty} d x\left\langle-x\left|\mathrm{e}^{-T H}\right| x\right\rangle
$$

Here the upper (lower) sign corresponds to the Dirichlet (Neumann) case. In the following the first term will be called the "direct" contribution, the second one the "indirect" one. In the path integral picture the second term represents the contribution to the heat kernel due to paths which involve a reflection at the boundary. The calculation of these whole line heat kernels involves the "doubled" potential

$$
\begin{aligned}
V(x) \rightarrow \tilde{V}(x) & =\theta(x) V(x)+\theta(-x) V(-x) \\
& =V_{+}(x)+\epsilon(x) V_{-}(x)
\end{aligned}
$$

where $\epsilon(x)$ is the "sign" function and $V_{ \pm}$indicates the even/odd part of the potential. In 28 this formalism was used to reproduce the known heat kernel coefficients for the half-space, as well as obtain two new ones, $a_{3}$ and $a_{7 / 2}$.

In the present paper, we present a more efficient and systematic approach along the same lines. The main improvements over [28] are the following:

1. We represent the sign function appearing in the doubled potential $\tilde{V}$ (1.16) through its Fourier transform,

$$
\epsilon(x)=\int_{-\infty}^{\infty} \frac{d p}{\pi p} \sin (p x)
$$

2. We use a path integral with antiperiodic boundary conditions for the calculation of the indirect contribution. 
The organization of this paper is as follows. In section 2 we explain a general method for calculating the indirect contribution to the half-line heat kernel,

$$
K_{\partial M}^{\text {ind }}(T):=\int_{0}^{\infty} d x\left\langle-x\left|\mathrm{e}^{-T H}\right| x\right\rangle
$$

We use the path integral formulation to derive a master formula for this indirect part which generalizes (1.12). We elaborate this master formula for the one and two point functions, i.e. the terms in $K_{\partial M}^{\text {ind }}$ involving one or two $V$ 's, and outline a procedure which allows one to obtain, recursively in the number of $V$ 's, a more explicit integral representation for this indirect part. Section 3 presents the more intricate procedure for calculating the direct part,

$$
K^{\operatorname{dir}}(T):=\int_{0}^{\infty} d x\left\langle x\left|\mathrm{e}^{-T H}\right| x\right\rangle=\int_{0}^{\infty} d x K_{M}^{\operatorname{dir}}(T, x)+K_{\partial M}^{\operatorname{dir}}(T)
$$

While the indirect part of the heat kernel contains only boundary terms, and will therefore be denoted by $K_{\partial M}^{\text {ind }}$, the direct one yields both a bulk contribution $K_{M}^{\text {dir }}$ and a boundary one $K_{\partial M}^{\mathrm{dir}}$. Again we demonstrate the efficiency of the method by an explicit treatment of the one and two point cases. We summarize our results in section 4. In appendix A we use our formalism for obtaining two more coefficients for the half-line heat kernel than were known before, $a_{4}$ and $a_{9 / 2}$. The generalization of the method from the half-line to a half-space is straightforward, and is presented in appendix $\mathrm{B}$.

\section{Heat kernel on the half-line: indirect contribution}

Let us thus reconsider the calculation of the heat kernel for the half-line (1.15). We will start with the indirect term, since, as will be seen, in the present approach it is easier to obtain than the direct one (this was different in the approach of [28]).

We first rewrite, using the symmetry $x \leftrightarrow-x$,

$$
\begin{aligned}
K_{\partial M}^{\text {ind }}(T) & =\int_{0}^{\infty} d x\left\langle-x\left|\mathrm{e}^{-T H}\right| x\right\rangle=\frac{1}{2} \int_{-\infty}^{\infty} d x\left\langle-x\left|\mathrm{e}^{-T H}\right| x\right\rangle \\
& =\frac{1}{2} \int_{A B C} \mathcal{D} x \exp \left[-\frac{1}{4 T} \int_{0}^{1} d \tau \dot{x}^{2}-T \int_{0}^{1} d \tau \tilde{V}(x(\tau))\right] \\
& =\frac{1}{4}\left\langle\mathrm{e}^{-T \int_{0}^{1} d \tau \tilde{V}(x(\tau))}\right\rangle_{A B C}
\end{aligned}
$$

where now the path integral is to be evaluated with antiperiodic boundary conditions, $x(1)=-x(0)$. Here in the last equation we have used the free antiperiodic path integral determinant which is easily obtained by, e.g., $\zeta$ - function regularization. One finds

$$
\int_{A B C} \mathcal{D} x \mathrm{e}^{-\frac{1}{4 T} \int_{0}^{1} d \tau \dot{x}^{2}}=\frac{1}{2}
$$

We also note that in the antiperiodic case there is no zero mode, no residual integration and therefore also no ambiguity in the Green's function. The appropriate worldline correlator is

$$
\begin{aligned}
\left\langle y^{\mu}(\tau) y^{\nu}(\sigma)\right\rangle & =-T \delta^{\mu \nu} G_{A}(\tau, \sigma), \\
G_{A}(\tau, \sigma) & =|\tau-\sigma|-\frac{1}{2}
\end{aligned}
$$


Note that $G_{A}$ is antiperiodic in both arguments.

We expand out the interaction exponential in (2.1). Using (1.16), (1.17), and Taylor expanding each $\tilde{V}^{(i)}\left(x\left(\tau_{i}\right)\right)$ at the boundary $x=0$, we can write

$$
\begin{aligned}
\tilde{V}^{(i)}\left(x_{i}\right) & =V_{+}^{(i)}\left(x_{i}\right)+\frac{1}{\pi} \int_{-\infty}^{\infty} \frac{d p_{i}}{p_{i}} \sin \left(p_{i} x_{i}\right) V_{-}^{(i)}\left(x_{i}\right) \\
& =\mathrm{e}^{x_{i} \partial_{i}} V_{+}^{(i)}(0)+\frac{1}{\pi i} \int_{\text {ev }} \frac{d p_{i}}{p_{i}} \mathrm{e}^{x_{i}\left(i p_{i}+\partial_{i}\right)} V_{-}^{(i)}(0)
\end{aligned}
$$

Here we denoted $x\left(\tau_{i}\right)=: x_{i}$, and we have introduced the abbreviation $\int_{\mathrm{ev}} d p_{i}$ for the integral $\int_{-\infty}^{\infty} d p_{i}$ with the understanding that only even powers of $p_{i}$ are to be kept in the integrand. Applying this procedure to the correlator (2.1), we obtain the following master formula for the indirect term,

$$
\begin{aligned}
K_{\partial M}^{\mathrm{ind}}= & \frac{1}{4} \sum_{n=0}^{\infty} \frac{(-T)^{n}}{n !} \int_{0}^{1} d \tau_{1} \cdots \int_{0}^{1} d \tau_{n} \exp \left[-\frac{T}{2} \sum_{i, j=1}^{n} G_{A}\left(\tau_{i}, \tau_{j}\right) D_{i}(p) D_{j}(p)\right] \\
& \times\left[V_{+}^{(1)}(0)+\frac{1}{\pi i} \int_{\mathrm{ev}} \frac{d p_{1}}{p_{1}} V_{-}^{(1)}(0)\right] \cdots\left[V_{+}^{(n)}(0)+\frac{1}{\pi i} \int_{\mathrm{ev}} \frac{d p_{n}}{p_{n}} V_{-}^{(n)}(0)\right]
\end{aligned}
$$

Here and in the following we define $D_{i}(p):=\partial_{i}+i p_{i}$, and it is understood that a $D_{i}(p)$ in the exponent acts as such on $V_{-}^{(i)}$, but reduces to $\partial_{i}$ when acting on $V_{+}^{(i)}$.

Although this master formula could be used as it stands to compute individual terms in the heat kernel expansion, it turns out to be advantageous to trade the parameters $p_{i}$ for parameters $s_{i}$, defined in the following way: first, we note that for the terms involving both $V_{+}$and $V_{-}$we can take the ordering to be $V_{-}^{(1)}, \ldots, V_{-}^{(m)}$ and $V_{+}^{(m+1)}, \ldots, V_{+}^{(n)}$. We then use the elementary identity

$$
\frac{1}{2 \pi i} \int_{-\infty}^{\infty} \frac{d p}{p} \mathrm{e}^{-a p^{2}}\left(\mathrm{e}^{i b p}-\mathrm{e}^{-i b p}\right)=\frac{b}{\sqrt{\pi a}} \int_{0}^{1} d s \mathrm{e}^{-\frac{b^{2}}{4 a} s^{2}}
$$

(valid for $a>0$ ) recursively with $p=p_{1}, \ldots, p=p_{m}$ to eliminate all the $p$ integrals and replace them by integrals $\int_{0}^{1} d s_{1} \cdots \int_{0}^{1} d s_{m}$.

We will now apply this formalism to the case of the one and two point functions.

\subsection{The one-point function}

The term with $n=1$ in the right hand side of the master formula (2.5) reads

$$
\begin{aligned}
K_{\partial M}^{\mathrm{ind}(1)}= & -\frac{T}{4} \int_{0}^{1} d \tau \exp \left[-\frac{T}{2} G_{A}(\tau, \tau)(\partial+i p)^{2}\right] \\
& \times\left[V_{+}(0)+\frac{1}{\pi i} \int_{\mathrm{ev}} \frac{d p}{p} V_{-}(0)\right]=: K_{\partial M+}^{\mathrm{ind}}+K_{\partial M-}^{\mathrm{ind}}
\end{aligned}
$$

Since $G_{A}(\tau, \tau)=-\frac{1}{2}$ there is no $\tau$ dependence in the one-point case, so that the $V_{+}$term becomes trivial,

$$
K_{\partial M+}^{\text {ind }}=-\frac{T}{4} \mathrm{e}^{\frac{T}{4} \partial^{2}} V_{+}(0)
$$


In the second term, we use (2.6) to transform it into

$$
\begin{aligned}
K_{\partial M-}^{\text {ind }} & =-\frac{T}{4} \mathrm{e}^{\frac{T}{4} \partial^{2}} \frac{1}{2 \pi i} \int_{\text {ev }} \frac{d p}{p} \mathrm{e}^{-\frac{T}{4} p^{2}}\left(\mathrm{e}^{i T \partial p / 2}-\mathrm{e}^{-i T \partial p / 2}\right) V_{-}(0) \\
& =-\frac{T^{\frac{3}{2}}}{4 \sqrt{\pi}} \int_{0}^{1} d s \mathrm{e}^{\left(1-s^{2}\right) \frac{T}{4} \partial^{2}} \partial V_{-}(0)
\end{aligned}
$$

Using (1.16) in reverse, and noting that even (odd) derivatives of $V_{-}\left(V_{+}\right)$vanish when evaluated at the boundary, the two terms can be recombined, and our final result for the indirect part of the one-point function becomes

$$
K_{\partial M}^{\operatorname{ind}(1)}(T)=-\frac{T}{4}\left(\mathrm{e}^{\frac{T}{4} \partial^{2}}+\sqrt{\frac{T}{\pi}} \int_{0}^{1} d s \mathrm{e}^{\left(1-s^{2}\right) \frac{T}{4} \partial^{2}} \partial\right) V(0)
$$

It is straightforward to expand the functional (2.10) and check that the coefficients of the expansions match those of the literature (see, e.g., [28]).

\subsection{The two-point function}

Proceeding to the terms quadratic in $V$, the simplest one in (2.5) is the one involving two $V_{+}$. It reads

$$
\begin{aligned}
K_{\partial M++}^{\mathrm{ind}}= & \frac{T^{2}}{8} \int_{0}^{1} d \tau_{1} \int_{0}^{1} d \tau_{2} \exp \left[\frac{T}{4}\left(\partial_{1}^{2}+\partial_{2}^{2}-4 G_{A 12} \partial_{1} \partial_{2}\right)\right] \\
& \times V_{+}^{(1)}(0) V_{+}^{(2)}(0)
\end{aligned}
$$

where we have abbreviated $G_{A i j}:=G_{A}\left(\tau_{i}, \tau_{j}\right)$. The terms involving both $V_{+}$and $V_{-}$ become, using again (2.6),

$$
\begin{aligned}
K_{\partial M+-}^{\mathrm{ind}}= & \frac{T^{2}}{8} \int_{0}^{1} d \tau_{1} \int_{0}^{1} d \tau_{2} \exp \left[\frac{T}{4}\left(\partial_{1}^{2}+\partial_{2}^{2}-4 G_{A 12} \partial_{1} \partial_{2}\right)\right] \\
& \times \frac{1}{\pi i} \int_{\mathrm{ev}} \frac{d p_{1}}{p_{1}} \exp \left[-\frac{T}{4} p_{1}^{2}+i T\left(\frac{1}{2} \partial_{1}-G_{A 12} \partial_{2}\right) p_{1}\right] V_{-}^{(1)}(0) V_{+}^{(2)}(0) \\
= & \frac{T^{\frac{5}{2}}}{8 \sqrt{\pi}} \int_{0}^{1} d \tau_{1} \int_{0}^{1} d \tau_{2} \exp \left[\frac{T}{4}\left(\partial_{1}^{2}+\partial_{2}^{2}-4 G_{A 12} \partial_{1} \partial_{2}\right)\right] \\
& \times\left(\partial_{1}-2 G_{A 12} \partial_{2}\right) \int_{0}^{1} d s_{1} \exp \left[-\frac{T}{4}\left(\partial_{1}-2 G_{A 12} \partial_{2}\right)^{2} s_{1}^{2}\right] \\
& \times V_{-}^{(1)}(0) V_{+}^{(2)}(0) \\
= & K_{\partial M-+}^{\mathrm{ind}}
\end{aligned}
$$

For the term with two $V_{-}$, we apply (2.6) first to $p_{1}$ and then to $p_{2}$. This yields

$$
\begin{aligned}
K_{\partial M--}^{\text {ind }}= & \frac{T^{2}}{8} \int_{0}^{1} d \tau_{1} \int_{0}^{1} d \tau_{2} \exp \left[\frac{T}{4}\left(\partial_{1}^{2}+\partial_{2}^{2}-4 G_{A 12} \partial_{1} \partial_{2}\right)\right] \\
& \times\left(\frac{1}{\pi i}\right)^{2} \int_{\mathrm{ev}} \frac{d p_{1}}{p_{1}} \int_{\mathrm{ev}} \frac{d p_{2}}{p_{2}} \exp \left\{T \left[-\frac{1}{4}\left(p_{1}^{2}+p_{2}^{2}\right)+G_{A 12} p_{1} p_{2}\right.\right.
\end{aligned}
$$




$$
\begin{gathered}
\left.\left.+i\left(\frac{1}{2} \partial_{1}-G_{A 12} \partial_{2}\right) p_{1}+i\left(\frac{1}{2} \partial_{2}-G_{A 12} \partial_{1}\right) p_{2}\right]\right\} \\
\times V_{-}^{(1)}(0) V_{-}^{(2)}(0) \\
=-\frac{T^{2}}{8 \pi} \int_{0}^{1} d \tau_{1} \int_{0}^{1} d \tau_{2} \exp \left[\frac{T}{4}\left(\partial_{1}^{2}+\partial_{2}^{2}-4 G_{A 12} \partial_{1} \partial_{2}\right)\right] \\
\times \int_{0}^{1} d s_{1} \frac{1}{\sqrt{1-4 G_{A 12}^{2} s_{1}^{2}}} \exp \left[-\frac{T}{4} s_{1}^{2}\left(\partial_{1}-2 G_{A 12} \partial_{2}\right)^{2}\right] \\
\times\left\{4 G_{A 12} \exp \left[-\frac{T}{4} \frac{\left(2\left(1-s_{1}^{2}\right) G_{A 12} \partial_{1}-\left(1-4 G_{A 12}^{2} s_{1}^{2}\right) \partial_{2}\right)^{2}}{1-4 G_{A 12}^{2} s_{1}^{2}}\right]\right. \\
+T\left(\partial_{1}-2 G_{A 12} \partial_{2}\right)\left(2\left(1-s_{1}^{2}\right) G_{A 12} \partial_{1}-\left(1-4 G_{A 12}^{2} s_{1}^{2}\right) \partial_{2}\right) \\
\left.\quad \times \int_{0}^{1} d s_{2} \exp \left[-s_{2}^{2} \frac{T}{4} \frac{\left(2\left(1-s_{1}^{2}\right) G_{A 12} \partial_{1}-\left(1-4 G_{A 12}^{2} s_{1}^{2}\right) \partial_{2}\right)^{2}}{1-4 G_{A 12}^{2} s_{1}^{2}}\right]\right\} \\
\times V_{-}^{(1)}(0) V_{-}^{(2)}(0)
\end{gathered}
$$

It is now easy to obtain from (2.11), 2.12), 2.13) any desired term in the derivative expansion of the two-point function. We give two examples. First, let us consider $K_{\partial M++}^{\text {ind }}$. Here the $\tau_{1,2}$ integrals can be done, for example, by expanding the exponential factor involving $G_{A 12}$, and using

$$
\int_{0}^{1} d \tau_{1} \int_{0}^{1} d \tau_{2} G_{A 12}^{n}=\frac{1}{2^{n}(n+1)}
$$

for $n$ even (remembering that odd powers of derivatives on $V_{+}(0)$ vanish). Resummation yields

$$
K_{\partial M++}^{\text {ind }}=\frac{T}{4} \mathrm{e}^{\frac{T}{4}\left(\partial_{1}^{2}+\partial_{2}^{2}\right)} \frac{\sinh \left(\frac{T}{2} \partial_{1} \partial_{2}\right)}{\partial_{1} \partial_{2}} V_{+}^{(1)} V_{+}^{(2)}
$$

Second, let us extract the leading contribution (as a power in $T$ ) from (2.13); such a contribution is proportional to $(\partial V(0))^{2}$. It is easy to see that the first term in braces in (2.13) does not contribute. The second one does, and yields

$$
\begin{aligned}
\frac{T^{3}}{8 \pi}(\partial V(0))^{2} & \int_{0}^{1} d \tau_{1} \int_{0}^{1} d \tau_{2} \int_{0}^{1} d s_{1} \frac{1-4 s_{1}^{2} G_{A 12}^{2}+4\left(1-s_{1}^{2}\right) G_{A 12}^{2}}{\sqrt{1-4 s_{1}^{2} G_{A 12}^{2}}} \\
& =\frac{T^{3}}{8 \pi}(\partial V(0))^{2} \int_{0}^{1} d \tau_{1} \int_{0}^{1} d \tau_{2}\left(\sqrt{1-4 G_{A 12}^{2}}+2 G_{A 12} \arcsin \left(2 G_{A 12}\right)\right) \\
& =T^{3} \frac{3}{64}(\partial V(0))^{2}
\end{aligned}
$$

This is one of the coefficients that in [28] needed to be fixed by means of a toy model. 


\section{Heat kernel on the half-line: direct contribution}

We proceed to the more involved calculation of the first ("direct") term of the heat kernel for the half-line (1.15). As in (2.1), we use the symmetry $x \leftrightarrow-x$ to make the $x$ integral run over the whole line,

$$
\begin{aligned}
K^{\operatorname{dir}}(T) & =\int_{0}^{\infty} d x K^{\operatorname{dir}}(T, x)=\frac{1}{2} \int_{-\infty}^{\infty} d x K^{\operatorname{dir}}(T, x) \\
& =\frac{1}{2} \int_{P B C} \mathcal{D} x \exp \left[-\frac{1}{4 T} \int_{0}^{1} d \tau \dot{x}^{2}-T \int_{0}^{1} d \tau \tilde{V}(x(\tau))\right]
\end{aligned}
$$

This integral is formally identical to the one without a boundary, eq. (1.5). Thus its calculation proceeds as in the whole line case, leading to the standard master formula (1.12) with $V(x)$ replaced by $\tilde{V}(x)$ :

$$
\begin{aligned}
K^{\operatorname{dir}}(T, x)= & (4 \pi T)^{-\frac{1}{2}} \sum_{n=0}^{\infty} \frac{(-T)^{n}}{n !} \int_{0}^{1} d \tau_{1} \cdots \int_{0}^{1} d \tau_{n} \\
& \times \exp \left[-\frac{T}{2} \sum_{i, j=1}^{n} G_{D, S}\left(\tau_{i}, \tau_{j}\right) \partial_{i} \partial_{j}\right] \prod_{i=1}^{n}\left[V_{+}^{(i)}(x)+\left(\epsilon(x) V_{-}(x)\right)^{(i)}\right]
\end{aligned}
$$

In the case of an even potential $\tilde{V}(x)=V_{+}(x)$ the further evaluation of this master formula would then also proceed as in the whole line case. Things get much more involved in the presence of $V_{-}(x)$, since the derivatives in (3.2) can also act on the $\epsilon(x)$ contained in $\tilde{V}(x)$, and produce $\delta$ functions and derivatives thereof. However such terms are boundary terms, therefore the complete bulk part of the heat kernel gets produced by the terms where all derivatives hit $V$ 's:

$$
\begin{aligned}
K_{M}^{\operatorname{dir}}(T, x)= & (4 \pi T)^{-\frac{1}{2}} \sum_{n=0}^{\infty} \frac{(-T)^{n}}{n !} \int_{0}^{1} d \tau_{1} \cdots \int_{0}^{1} d \tau_{n} \\
& \times \exp \left[-\frac{T}{2} \sum_{i, j=1}^{n} G_{D, S}\left(\tau_{i}, \tau_{j}\right) \partial_{i} \partial_{j}\right] \prod_{i=1}^{n}\left[V_{+}^{(i)}(x)+\epsilon(x) V_{-}^{(i)}(x)\right]
\end{aligned}
$$

Here our notation is meant to convey that the derivative $\partial_{i}$ acts only on $V^{(i)}$, not on $\epsilon(x)$. In this bulk part one is now free to take $x$ positive and to replace $V_{+}^{(i)}(x)+\epsilon(x) V_{-}^{(i)}(x)$ by $V^{(i)}(x)$.

For the explicit evaluation of the boundary part, we use again the Fourier representation of the $\epsilon$ function (1.17). This allows us to rewrite (3.2) as

$$
\begin{aligned}
K^{\operatorname{dir}}(T, x)= & (4 \pi T)^{-\frac{1}{2}} \sum_{n=0}^{\infty} \frac{(-T)^{n}}{n !} \int_{0}^{1} d \tau_{1} \cdots \int_{0}^{1} d \tau_{n} \\
& \times \exp \left[-\frac{T}{2} \sum_{i, j=1}^{n} G_{D, S}\left(\tau_{i}, \tau_{j}\right) D_{i}(p) D_{j}(p)\right] \\
& \times \prod_{k=1}^{n}\left[V_{+}^{(k)}(x)+\frac{1}{\pi i} \int_{\text {ev }} \frac{d p_{k}}{p_{k}} \mathrm{e}^{i p_{k} x} V_{-}^{(k)}(x)\right]
\end{aligned}
$$


Now (3.4) and (3.3) differ only in the exponential prefactor. Rewriting this difference as the integral of a total derivative in a new variable $w$, and integrating over $x$, we obtain the following master formula for $K_{\partial M}^{\mathrm{dir}}$ :

$$
\begin{aligned}
K_{\partial M}^{\operatorname{dir}}(T)= & \frac{1}{2} \int_{-\infty}^{\infty} d x\left(K^{\operatorname{dir}}(T, x)-K_{M}^{\operatorname{dir}}(T, x)\right) \\
= & \frac{1}{2}(4 \pi T)^{-\frac{1}{2}} \sum_{n=0}^{\infty} \frac{(-T)^{n}}{n !} \int_{0}^{1} d \tau_{1} \cdots \int_{0}^{1} d \tau_{n} \int_{-\infty}^{\infty} d x \\
& \times \int_{0}^{1} d w \frac{\partial}{\partial w} \exp \left[-\frac{T}{2} \sum_{i, j=1}^{n} G_{D, S}\left(\tau_{i}, \tau_{j}\right) D_{i}(w p) D_{j}(w p)\right] \\
& \times \prod_{k=1}^{n}\left[V_{+}^{(k)}(x)+\frac{1}{\pi i} \int_{\text {ev }} \frac{d p_{k}}{p_{k}} \mathrm{e}^{i p_{k} x} V_{-}^{(k)}(x)\right]
\end{aligned}
$$

Since this difference contains only boundary terms, it is justified to Taylor expand $V_{ \pm}(x)$ with respect to the boundary, $V_{ \pm}(x)=\mathrm{e}^{x \partial} V_{ \pm}(0)$. The $x$ integral can then be done, and yields a $\delta$ function involving the various $p_{k}$ 's and $\partial_{k}$ 's. The $w$-derivative always cancels one of the (spurious) poles in the $p_{k}$ 's, after which one can use the $\delta$ function to eliminate the corresponding $p_{k}$ integral. After this one first does the remaining $p_{k}$ integrals, then the $w$ integral, and finally the $\tau_{i}$ integrals. The latter ones will be of Selberg type. Here it should also be mentioned that, due to the fact that the string-inspired Green's function preserves the worldline translation invariance, in the SI scheme it is always possible to eliminate one of the $\tau$ - integrals trivially, i.e. by setting $\tau_{n}=0$. This is generally not the case for the DBC scheme.

As for the indirect contribution, we will demonstrate the procedure by an explicit calculation of the one and two point contributions.

\subsection{The one-point function}

At the one-point level, the bulk master formula (3.3) gives

$$
K_{M}^{\operatorname{dir}(1)}(T, x)=-T(4 \pi T)^{-\frac{1}{2}} \int_{0}^{1} d \tau \mathrm{e}^{-\frac{T}{2} G(\tau, \tau) \partial^{2}} V(x)
$$

where $G(\tau, \sigma)$ stands for either Green's function. For the boundary term we get from (3.5), after expanding $V_{-}(x)$ at $x=0$,

$$
\begin{aligned}
K_{\partial M}^{\operatorname{dir}(1)}(T)= & -\frac{T}{2}(4 \pi T)^{-\frac{1}{2}} \int_{0}^{1} d \tau \int_{\mathrm{ev}} \frac{d p}{i \pi p} \int_{-\infty}^{\infty} d x \mathrm{e}^{(i p+\partial) x} \\
& \times \int_{0}^{1} d w \partial_{w} \mathrm{e}^{-\frac{T}{2} G(\tau, \tau)(\partial+i w p)^{2}} V_{-}(0) \\
= & -\frac{T}{2}(4 \pi T)^{-\frac{1}{2}} \int_{0}^{1} d \tau \int_{\mathrm{ev}} \frac{d p}{i \pi p} 2 \pi \delta(p-i \partial) \\
& \times(-T G(\tau, \tau)) i p \int_{0}^{1} d w(\partial+i w p) \mathrm{e}^{-\frac{T}{2} G(\tau, \tau)(\partial+i w p)^{2}} V_{-}(0)
\end{aligned}
$$




$$
\begin{aligned}
= & T^{2}(4 \pi T)^{-\frac{1}{2}} \int_{0}^{1} d \tau G(\tau, \tau) \int_{0}^{1} d w(1-w) \\
& \times \mathrm{e}^{-\frac{T}{2} G(\tau, \tau)(1-w)^{2} \partial^{2}} \partial V_{-}(0) \\
= & T(4 \pi T)^{-\frac{1}{2}} \int_{0}^{1} d \tau \partial^{-1}\left(1-e^{-\frac{T}{2} G(\tau, \tau) \partial^{2}}\right) V_{-}(0)
\end{aligned}
$$

The essential point to be noted is that the total derivative in $w$ has led to the appearance of a factor of $p$ in the numerator which cancels the one in the denominator, making it possible to apply the $\delta$ function coming from the $x$ integral.

Note also that, except the leading order, all terms in the expansion of both the boundary part and the bulk part of the one-point function are dependent upon the choice of worldline Green's function. However, let us verify that the complete (integrated) one-point function is scheme-independent. Combining (3.6) and (3.7) we can write this as

$$
\begin{aligned}
K^{\operatorname{dir}(1)}(T)=-T(4 \pi T)^{-1 / 2} \int_{0}^{1} d \tau & {\left[\int_{0}^{\infty} d x e^{-\frac{T}{2} G(\tau, \tau) \partial^{2}} V(x)\right.} \\
& \left.+\partial^{-1}\left(e^{-\frac{T}{2} G(\tau, \tau) \partial^{2}}-1\right) V(0)\right]
\end{aligned}
$$

Using partial integration it is thus easy to see that the latter reduces to

$$
K^{\operatorname{dir}(1)}(T)=-T(4 \pi T)^{-1 / 2} \int_{0}^{\infty} d x V(x)
$$

which is manifestly independent of the choice of Green's function.

\subsection{The two-point function}

At two points, the bulk master formula (3.3) yields

$$
\begin{aligned}
K_{M}^{\operatorname{dir}(2)}(T, x)= & (4 \pi T)^{-\frac{1}{2}} \frac{T^{2}}{2 !} \int_{0}^{1} d \tau_{1} \int_{0}^{1} d \tau_{2} \\
& \times \exp \left[-\frac{T}{2} \sum_{i, j=1}^{2} G_{i j} \partial_{i} \partial_{j}\right] V^{(1)}(x) V^{(2)}(x)
\end{aligned}
$$

The boundary master formula (3.5) now gives three contributions,

$$
K_{\partial M}^{\mathrm{dir}(2)}=K_{\partial M+-}^{\mathrm{dir}}+K_{\partial M-+}^{\mathrm{dir}}+K_{\partial M--}^{\mathrm{dir}}
$$

where $K_{\partial M+-}^{\mathrm{dir}}=K_{\partial M-+}^{\mathrm{dir}}$. The mixed term is the simpler one to calculate:

$$
\begin{aligned}
K_{\partial M-+}^{\mathrm{dir}}= & \frac{T^{2}}{4}(4 \pi T)^{-1 / 2} \int_{-\infty}^{\infty} d x \int_{0}^{1} d \tau_{1} \int_{0}^{1} d \tau_{2} \mathrm{e}^{-\frac{T}{2} G_{22} \partial_{2}^{2}} \\
& \times\left[\mathrm{e}^{-\frac{T}{2}\left(G_{11}\left(\partial_{1}+i p_{1}\right)^{2}+2 G_{12}\left(\partial_{1}+i p_{1}\right) \partial_{2}\right)}-\mathrm{e}^{-\frac{T}{2}\left(G_{11} \partial_{1}^{2}+2 G_{12} \partial_{1} \partial_{2}\right)}\right] \\
& \times \int_{\text {ev }} \frac{d p_{1}}{i \pi p_{1}} \mathrm{e}^{i p_{1} x} V_{-}^{(1)}(x) V_{+}^{(2)}(x)
\end{aligned}
$$




$$
\begin{aligned}
= & \frac{T^{2}}{4}(4 \pi T)^{-1 / 2} \int_{0}^{1} d \tau_{1} \int_{0}^{1} d \tau_{2} \mathrm{e}^{-\frac{T}{2} G_{22} \partial_{2}^{2}} \\
& \times \int_{0}^{1} d w \partial_{w} \mathrm{e}^{-\frac{T}{2}\left(G_{11}\left(\partial_{1}+i w p_{1}\right)^{2}+2 G_{12}\left(\partial_{1}+i w p_{1}\right) \partial_{2}\right)} \\
& \times \int_{\mathrm{ev}} \frac{d p_{1}}{i \pi p_{1}} \int_{-\infty}^{\infty} d x \mathrm{e}^{\left(i p_{1}+\partial_{1}+\partial_{2}\right) x} V_{-}^{(1)}(0) V_{+}^{(2)}(0) \\
= & \frac{T^{2}}{4}(4 \pi T)^{-1 / 2} \int_{0}^{1} d \tau_{1} \int_{0}^{1} d \tau_{2} \mathrm{e}^{-\frac{T}{2} G_{22} \partial_{2}^{2}} \\
& \times \int_{0}^{1} d w\left(-i T p_{1}\right)\left(G_{11}\left(\partial_{1}+i w p_{1}\right)+G_{12} \partial_{2}\right) \\
& \times \mathrm{e}^{-\frac{T}{2}\left(G_{11}\left(\partial_{1}+i w p_{1}\right)^{2}+2 G_{12}\left(\partial_{1}+i w p_{1}\right) \partial_{2}\right)} \\
& \times \int_{\mathrm{ev}} \frac{d p_{1}}{i \pi p_{1}} 2 \pi \delta\left(p_{1}-i \partial_{1}-i \partial_{2}\right) V_{-}^{(1)}(0) V_{+}^{(2)}(0) \\
= & -\frac{T^{3}}{2}(4 \pi T)^{-1 / 2} \int_{0}^{1} d \tau_{1} \int_{0}^{1} d \tau_{2} \mathrm{e}^{-\frac{T}{2} G_{22} \partial_{2}^{2}} \\
& \times \int_{0}^{1} d w\left(G_{11}\left[(1-w) \partial_{1}-w \partial_{2}\right]+G_{12} \partial_{2}\right) \\
& \times \mathrm{e}^{-\frac{T}{2}\left(G_{11}\left[(1-w) \partial_{1}-w \partial_{2}\right]^{2}+2 G_{12}\left[(1-w) \partial_{1}-w \partial_{2}\right] \partial_{2}\right)} V_{-}^{(1)}(0) V_{+}^{(2)}(0)
\end{aligned}
$$

The case of $K_{\partial M--}^{\mathrm{dir}}$ is somewhat more laborious:

$$
\begin{aligned}
K_{\partial M--}^{\mathrm{dir}}= & \frac{T^{2}}{4}(4 \pi T)^{-1 / 2} \int_{0}^{1} d \tau_{1} \int_{0}^{1} d \tau_{2} \\
& \times \int_{0}^{1} d w \partial_{w} \mathrm{e}^{-\frac{T}{2} \sum_{i, j=1}^{2} G_{i j}\left(\partial_{i}+i w p_{i}\right)\left(\partial_{j}+i w p_{j}\right)} \\
& \times \int_{\mathrm{ev}} \frac{d p_{1}}{i \pi p_{1}} \int_{\mathrm{ev}} \frac{d p_{2}}{i \pi p_{2}} 2 \pi \delta\left(p_{1}+p_{2}-i \partial_{1}-i \partial_{2}\right) V_{-}^{(1)}(0) V_{-}^{(2)}(0) \\
= & (-i T) \frac{T^{2}}{4}(4 \pi T)^{-1 / 2} \int_{0}^{1} d \tau_{1} \int_{0}^{1} d \tau_{2} \int_{0}^{1} d w \\
& \times\left[G_{11} p_{1}\left(\partial_{1}+i w p_{1}\right)+G_{22} p_{2}\left(\partial_{2}+i w p_{2}\right)\right. \\
& \left.+G_{12}\left(p_{1} \partial_{2}+p_{2} \partial_{1}+2 i w p_{1} p_{2}\right)\right] \mathrm{e}^{-\frac{T}{2} \sum_{i, j=1}^{2} G_{i j}\left(\partial_{i}+i w p_{i}\right)\left(\partial_{j}+i w p_{j}\right)} \\
& \times \int_{\mathrm{ev}} \frac{d p_{1}}{i \pi p_{1}} \int_{\mathrm{ev}} \frac{d p_{2}}{i \pi p_{2}} 2 \pi \delta\left(p_{1}+p_{2}-i \partial_{1}-i \partial_{2}\right) V_{-}^{(1)}(0) V_{-}^{(2)}(0)
\end{aligned}
$$

Using the symmetry $1 \leftrightarrow 2$, the expression in square brackets can be simplified to

$$
2 p_{2}\left[G_{22} \partial_{2}+G_{22} i w p_{2}+G_{12} \partial_{1}+i G_{12} w p_{1}\right]
$$

so that the $p_{2}$ pole cancels and we may use the $\delta$ function to eliminate $p_{2}$ altogether. This leads to

$$
K_{\partial M--}^{\mathrm{dir}}=-T^{3}(4 \pi T)^{-1 / 2} \int_{0}^{1} d \tau_{1} \int_{0}^{1} d \tau_{2} \int_{0}^{1} d w \int_{\mathrm{ev}} \frac{d p_{1}}{i \pi p_{1}}
$$




$$
\begin{aligned}
& \times\left[G_{22}\left(\partial_{2}-w\left(i p_{1}+\partial_{1}+\partial_{2}\right)\right)+G_{12}\left(\partial_{1}+i w p_{1}\right)\right] \\
& \times \exp \left\{-\frac{T}{2}\left[G_{11}\left(\partial_{1}+i w p_{1}\right)^{2}+G_{22}\left(\partial_{2}-w\left(i p_{1}+\partial_{1}+\partial_{2}\right)\right)^{2}\right.\right. \\
& \left.\left.\quad+2 G_{12}\left(\partial_{1}+i w p_{1}\right)\left(\partial_{2}-w\left(i p_{1}+\partial_{1}+\partial_{2}\right)\right)\right]\right\} \\
& \times V_{-}^{(1)}(0) V_{-}^{(2)}(0)
\end{aligned}
$$

Individual terms in the derivative expansion of this two-point function are now obtained by expanding out the exponential factors in eqs. (3.10), (3.12), (3.15). Note that in the case of (3.12) this immediately leads to a polynomial $w$ integral. For (3.15) we have to remember the fact that $\partial^{n} V_{-}(0)=0$ for even $n$; it is then easily seen that all surviving terms in this expansion carry a factor of $p_{1}$. The $p_{1}$ integral then becomes a simple Gaussian one, and doing it one gets a $w$ integral which is polynomial. It is only for the final $\tau_{1,2}$ integrals that one has to specify the Green's function $G\left(\tau_{1}, \tau_{2}\right)$. As we have seen already in the one-point case, the coefficients of a given term will generally depend on the choice of the worldline Green's function; the equivalence of the results obtained with different worldline Greens' functions can only be seen after adding up bulk and boundary terms, and performing certain integration by parts. However, since bulk terms generally have an even number of derivatives, the boundary terms involved in integration by parts have an odd one. It is therefore clear that those terms in $K_{\partial M}^{\mathrm{dir}}$ with an even number of derivatives are always independent of the choice of the worldline Green's function.

Let us exemplify all this by extracting the lowest order term in the two-point function, i.e. the coefficient of $\partial V_{-}(0) \partial V_{-}(0)$. In the indirect sector only 3.15 contributes to it. Collecting from (3.15) all the terms involving $\partial_{1} V_{-}^{(1)}(0) \partial_{2} V_{-}^{(2)}(0)$, performing the integral over $p_{1}$, which is now Gaussian, and integrating out the auxiliary variable $w$ one obtains

$$
T^{7 / 2}(4 \pi T)^{-1 / 2}(\partial V(0))^{2} \frac{1}{3 \sqrt{2 \pi}} \int_{0}^{1} d \tau_{1} \int_{0}^{1} d \tau_{2}\left(2 G_{12}-G_{11}-G_{22}\right)^{3 / 2}
$$

Written in this form it is easy to see that the integrand does not depend on the choice of worldline Green's functions. ${ }^{2}$ Translation invariance of the SI propagator allows one to set $\tau_{2}=0$, leaving a single integral

$$
\int_{0}^{1} d \tau_{1} \int_{0}^{1} d \tau_{2}\left(2 G_{12}-G_{11}-G_{22}\right)^{3 / 2}=2^{3 / 2} \int_{0}^{1} d \tau[\tau(1-\tau)]^{3 / 2}=\frac{3 \sqrt{2} \pi}{2^{6}}
$$

\footnotetext{
${ }^{2}$ Worldline Green's functions are usually defined using a linear constraint $\int_{0}^{1} d \tau \rho(\tau) y(\tau)=0$, with $\int_{0}^{1} d \tau \rho(\tau) y(\tau)=1$ (see [7, 6). This definition includes as special cases the "string inspired" method (with nonvanishing coincidence limit) identified by $\rho=1$, for which $\tilde{G}_{S}(\tau-\sigma)=|\tau-\sigma|-(\tau-\sigma)^{2}-\frac{1}{6}$, and the DBC one identified by $\rho(\tau)=\delta(\tau)$. The Green's function corresponding to a generic $\rho$ is thus related to the string inspired Green's function given in (1.9) by the relation $G(\tau, \sigma)=G_{S}(\tau-\sigma)-\int_{0}^{1} d \lambda G_{S}(\tau-$ $\lambda) \rho(\lambda)-\int_{0}^{1} d \lambda G_{S}(\sigma-\lambda) \rho(\lambda)+\int_{0}^{1} d \lambda \int_{0}^{1} d \mu G_{S}(\lambda-\mu) \rho(\lambda) \rho(\mu)$. Using this relation it is simple to show the scheme-independence of 3.16. Also, it is clear that the coincidence limit can be dropped from the string inspired propagator; in general, dropping such a limit just amounts to dropping total derivative terms from the heat kernel coefficients.
} 
The indirect contribution of this term to $a_{7 / 2}$ becomes

$$
\frac{T^{7 / 2}}{(4 \pi T)^{1 / 2}} \frac{\sqrt{\pi}}{2} \frac{1}{2^{5}}(\partial V(0))^{2}
$$

that is in accordance with [28].

\section{Conclusions}

This work should be seen as a further step towards making the worldline formalism useful for analytic calculations of effective actions in the presence of nontrivial boundary conditions. The case of a scalar field on a half-space with Dirichlet or Neumann conditions is the natural starting point for such a program. While in [28] the problem of applying the worldline formalism to this case had been solved in principle, the solution was not yet optimized from a technical point of view. The various improvements which we have implemented in the present work lead to a much simpler algorithm, which we consider very promising for future generalizations of the analytic worldline approach to the calculation of effective actions involving more general fields, as well as more general boundaries and types of boundary conditions. For example, in the case of general boundaries in curved spaces, although it is reasonable to think that the image charges could increase in number and even become a continuous distribution, it is also plausible that, for points sufficiently close to a smooth boundary and in suitable coordinates, the main contributions arise from a single image charge. On the other hand for specific geometries, for example a suitable curved boundary in flat space, one might find a neat set up of image charges that could solve the problem exactly.

For the case of the half-line, prior to the work of [28] the heat kernel for an arbitrary potential $V(x)$ was known only up to $a_{5 / 2}$, to our knowledge. In 28] the coefficients $a_{3}$, $a_{7 / 2}$ were obtained, although this required already a substantial computational effort. As we show in appendix A, our improved formalism makes it relatively easy to push this calculation to $a_{9 / 2}$. Moreover, the algorithms developed in section 2 for the indirect and in section 3 for the direct contributions to the heat kernel with Dirichlet or Neumann boundary conditions can be easily computerized. This should allow one to obtain many more coefficients beyond the known ones. In addition, one may also include more general types of boundary conditions in the present formalism [29].

It must be emphasized that the results for the local heat kernel, i.e. the heat kernel diagonal $K(T ; x, x)$, generally depend on the worldline Green's function used in the calculation of the direct part. Only when used with the 'DBC' Green's function $G_{D}$ our algorithm yields the standard heat kernel. The use of the 'string-inspired' or other worldline Green's functions will yield a result which is different locally, and agrees with the standard heat kernel one only after integration over the bulk, and summation of bulk and boundary contributions,

$$
\int_{0}^{\infty} d x K_{M}(T, x)+K_{\partial M}(T)
$$

On the other hand, the calculation of $K^{\text {dir }}$ becomes simpler with the string-inspired Green's function, which is therefore preferable for effective action calculations where usually only the total effective action is relevant. 


\section{Acknowledgments}

F.B., P.P. and C.S. thank the Albert-Einstein Institute, Potsdam, for hospitality. O.C. is grateful to the Instituto de Física y Matemáticas of the UMSNH at Morelia for hospitality and partial support. P.P. and C.S. are grateful to INFN and Dipartimento di Fisica of the Università di Bologna for hospitality. The work of F.B. and O.C. was partly supported by the Italian MIUR-PRIN contract 20075ATT78. The work of P.P. was partly supported by PIP 6160, UNLP proj. 11/X381 and DAAD.

\section{A. Half-line heat kernel coefficients $a_{4}$ and $a_{9 / 2}$}

The indirect contributions to the $a_{4}$ and $a_{9 / 2}$ coefficients can be obtained with the method described in section 2 and read

$$
\begin{aligned}
a_{4}^{\text {ind }}=- & \frac{1}{4} V^{2} \partial V(0)+\frac{1}{6} \partial V \partial^{2} V(0)+\frac{1}{12} V \partial^{3} V(0)-\frac{1}{5 !} \partial^{5} V(0) \\
a_{9 / 2}^{\text {ind }}=\frac{\sqrt{\pi}}{2} & {\left[-\frac{1}{3 ! 2^{6}} \partial^{6} V(0)+\frac{1}{4 !} V^{4}(0)-\frac{3}{2^{4}}(\partial V)^{2} V(0)-\frac{1}{2^{3}} V^{2} \partial^{2} V(0)\right.} \\
& \left.+\frac{1}{2^{5}} V \partial^{4} V(0)+\frac{5}{3 \cdot 2^{5}}\left(\partial^{2} V(0)\right)^{2}+\frac{5}{2^{6}} \partial^{3} V \partial V(0)\right]
\end{aligned}
$$

The direct contributions to the same coefficients are obtained with the method of section (3) and their boundary contributions read

$$
\begin{aligned}
\left.a_{4}^{\text {dir }}\right|_{\partial M} & =\alpha_{1} V^{2} \partial V(0)+\alpha_{2} \partial V \partial^{2} V(0)+\alpha_{3} V \partial^{3} V(0)+\alpha_{4} \partial^{5} V(0) \\
a_{9 / 2}^{\text {dir }} & =\frac{\sqrt{\pi}}{2}\left[\beta_{1}(\partial V)^{2} V(0)+\beta_{2} \partial^{3} V \partial V(0)\right]
\end{aligned}
$$

where

$$
\begin{aligned}
& \alpha_{1}=\frac{1}{4} \int_{0}^{1} d \tau_{1} G_{11}= \begin{cases}0 & (S I) \\
-\frac{1}{2^{2} \cdot 3} & (D B C)\end{cases} \\
& \alpha_{2}=\frac{1}{2} \int_{0}^{1} d \tau_{1} \int_{0}^{1} d \tau_{2}\left[\frac{1}{2} G_{11} G_{22}+\frac{1}{4} G_{11}^{2}+G_{12}^{2}-G_{11} G_{12}\right]= \begin{cases}\frac{2}{5 !} & (S I) \\
\frac{4}{5 !} & (D B C)\end{cases} \\
& \alpha_{3}=\frac{1}{8} \int_{0}^{1} d \tau_{1} G_{11}^{2}= \begin{cases}0 & (S I) \\
\frac{2}{5 !} & (D B C)\end{cases} \\
& \alpha_{4}=\frac{1}{3 ! 2^{3}} \int_{0}^{1} d \tau_{1} G_{11}^{3}= \begin{cases}0 & (S I) \\
-\frac{3 !}{7 !} & (D B C)\end{cases}
\end{aligned}
$$


and

$$
\begin{aligned}
& \beta_{1}=-\frac{\sqrt{2}}{3 \pi} \int_{0}^{1} d \tau_{1} \int_{0}^{1} d \tau_{2}\left(2 G_{12}-G_{11}-G_{22}\right)^{3 / 2}=-\frac{1}{2^{5}} \\
& \beta_{2}=\frac{2 \sqrt{2}}{15 \pi} \int_{0}^{1} d \tau_{1} \int_{0}^{1} d \tau_{2}\left(2 G_{12}-G_{11}-G_{22}\right)^{5 / 2}=\frac{1}{2^{6} \cdot 3}
\end{aligned}
$$

that are scheme-independent. We have checked that the complete $a_{4}$ coefficient is independent of the propagator chosen (SI or DBC), as expected.

\section{B. Heat kernel on the half-space}

Let us consider the flat space $\mathcal{M}=\mathbb{R}_{+} \times \mathbb{R}^{D-1}$ with coordinates $X^{\beta}:=\left(x^{0}, x^{b}\right):=\left(x^{0}, \vec{x}\right)$, where $0 \leq x^{0}<\infty$ and $\vec{x} \in \mathbb{R}^{D-1}$. The generalization of our algorithm from the half-line to the half-space is straightforward, starting with the generalization of $\tilde{V}$,

$$
\begin{aligned}
\tilde{V}(X) & :=\theta\left(x^{0}\right) V\left(x^{0}, \vec{x}\right)+\theta\left(-x^{0}\right) V\left(-x^{0}, \vec{x}\right) \\
& =V_{+}(X)+\epsilon\left(x^{0}\right) V_{-}(X)
\end{aligned}
$$

We will therefore give the final master formulas generalizing eqs. (2.5) and (3.4), (3.3), (3.5) and list the short-time expansion of the heat kernel in the half space. In the following it should now be understood that $D_{i}(p) \cdot D_{j}(p)=\sum_{\beta=0}^{D-1} D_{i}^{\beta}(p) D_{j}^{\beta}(p)$, and that only the zeroeth component of $D_{i}(p)$ has a $p$ - part (when acting on $V_{-}$), i.e. $D_{i}^{\beta}(p)=\partial_{i}^{\beta}+\delta^{\beta 0} i p_{i}$. Hence,

$$
\begin{aligned}
K_{\partial M}^{\text {ind }}= & \frac{1}{4}(4 \pi T)^{-\frac{D-1}{2}} \sum_{n=0}^{\infty} \frac{(-T)^{n}}{n !} \int_{0}^{1} d \tau_{1} \cdots \int_{0}^{1} d \tau_{n} \int d^{D-1} x \\
& \times \exp \left[-\frac{T}{2} \sum_{i, j=1}^{n}\left(G_{D, S}\left(\tau_{i}, \tau_{j}\right) \vec{\partial}_{i} \cdot \vec{\partial}_{j}+G_{A}\left(\tau_{i}, \tau_{j}\right) D_{i}^{0}(p) D_{j}^{0}(p)\right)\right] \\
& \times \prod_{k=1}^{n}\left[V_{+}^{(k)}(0, \vec{x})+\frac{1}{\pi i} \int_{\mathrm{ev}} \frac{d p_{k}}{p_{k}} V_{-}^{(k)}(0, \vec{x})\right]
\end{aligned}
$$

is the master formula for the indirect term, whereas

$$
\begin{aligned}
K^{\operatorname{dir}}(T, X)= & (4 \pi T)^{-\frac{D}{2}} \sum_{n=0}^{\infty} \frac{(-T)^{n}}{n !} \int_{0}^{1} d \tau_{1} \cdots \int_{0}^{1} d \tau_{n} \\
& \times \exp \left[-\frac{T}{2} \sum_{i, j=1}^{n} G_{D, S}\left(\tau_{i}, \tau_{j}\right) D_{i}(p) \cdot D_{j}(p)\right] \\
& \times \prod_{k=1}^{n}\left[V_{+}^{(k)}(X)+\frac{1}{\pi i} \int_{\mathrm{ev}} \frac{d p_{k}}{p_{k}} \mathrm{e}^{i p_{k} x^{0}} V_{-}^{(k)}(X)\right] \\
K_{M}^{\operatorname{dir}}(T, X)= & (4 \pi T)^{-\frac{D}{2}} \sum_{n=0}^{\infty} \frac{(-T)^{n}}{n !} \int_{0}^{1} d \tau_{1} \cdots \int_{0}^{1} d \tau_{n}
\end{aligned}
$$




$$
\begin{aligned}
& \times \exp \left[-\frac{T}{2} \sum_{i, j=1}^{n} G_{D, S}\left(\tau_{i}, \tau_{j}\right) \partial_{i} \cdot \partial_{j}\right] \prod_{i=1}^{n} V^{(i)}(X), \quad\left(x^{0}>0\right) \\
K_{\partial M}^{\operatorname{dir}}(T)= & \frac{1}{2}(4 \pi T)^{-\frac{D}{2}} \sum_{n=0}^{\infty} \frac{(-T)^{n}}{n !} \int_{0}^{1} d \tau_{1} \cdots \int_{0}^{1} d \tau_{n} \int_{-\infty}^{\infty} d x^{0} \int d^{D-1} x \\
& \times \int_{0}^{1} d w \frac{\partial}{\partial w} \exp \left[-\frac{T}{2} \sum_{i, j=1}^{n} G_{D, S}\left(\tau_{i}, \tau_{j}\right) D_{i}(w p) \cdot D_{j}(w p)\right] \\
& \times \prod_{k=1}^{n}\left[V_{+}^{(k)}(X)+\frac{1}{\pi i} \int_{\mathrm{ev}} \frac{d p_{k}}{p_{k}} \mathrm{e}^{i p_{k} x^{0}} V_{-}^{(k)}(X)\right]
\end{aligned}
$$

are respectively the master formulas for the complete local direct term, its bulk part, and its integrated boundary part.

Finally we report the coefficients, up to order $9 / 2$, of the short-time expansion for the heat-kernel trace, computed using the above formulas with the SI Green's function

$$
K_{S}(T)=(4 \pi T)^{-\frac{D}{2}} \sum_{n \in \mathbb{N} / 2} a_{n} T^{n}
$$

with

$$
\begin{aligned}
a_{0}= & \int_{M} 1 \\
a_{1 / 2}= & \frac{\sqrt{\pi}}{2} \int_{\partial M}(\mp 1) \\
a_{1}= & \int_{M}(-V) \\
a_{3 / 2}= & \frac{\sqrt{\pi}}{2} \int_{\partial M}( \pm V) \\
a_{2}= & \int_{M} \frac{1}{2 !} V^{2}+\int_{\partial M} \frac{1}{2 !}\left( \pm \partial_{0} V\right) \\
a_{5 / 2}= & \frac{\sqrt{\pi}}{2} \int_{\partial M} \frac{1}{2 !}(\mp)\left(V^{2}-\frac{1}{2} \partial_{0}^{2} V\right) \\
a_{3}= & \int_{M} \frac{1}{3 !}\left(-V^{3}-\frac{1}{2}\left(\partial_{\beta} V\right)^{2}\right)+\int_{\partial M} \frac{1}{3 !}(\mp)\left(3 V \partial_{0} V-\frac{1}{2} \partial_{0}^{3} V\right) \\
a_{7 / 2}= & \frac{\sqrt{\pi}}{2} \int_{\partial M} \frac{1}{3 !}\left[\mp\left(-V^{3}+\frac{3}{2} V \partial_{0}^{2} V-\frac{1}{2}(\vec{\partial} V)^{2}-\frac{3}{16} \partial_{0}^{4} V\right)+\{-5\} \frac{3}{7}\left(\partial_{0} V\right)^{2}\right] \\
a_{4}= & \int_{M} \frac{1}{4 !}\left[V^{4}+2 V\left(\partial_{\beta} V\right)^{2}+\frac{1}{5}\left(\partial_{\beta} \partial_{\gamma} V\right)^{2}\right] \\
& +\int_{\partial M} \frac{1}{4 !}\left[\mp\left(-6 V^{2} \partial_{0} V+2 V \partial_{0}^{3} V-2 \vec{\partial} V \cdot \vec{\partial} \partial_{0} V-\frac{1}{5} \partial_{0}^{5} V\right)+\left\{\begin{array}{l}
-9 \\
11
\end{array}\right\} \frac{2}{5} \partial_{0} V \partial_{0}^{2} V\right]
\end{aligned}
$$




$$
\begin{aligned}
a_{9 / 2}=\frac{\sqrt{\pi}}{2} \int_{\partial M} \frac{1}{4 !}\left[\mp \left(V^{4}-3 V^{2} \partial_{0}^{2} V+2 V(\vec{\partial} V)^{2}+\frac{1}{5}\left(\partial_{b} \partial_{c} V\right)^{2}+\frac{3}{4} V \partial_{0}^{4} V\right.\right. \\
\left.\quad-\vec{\partial} V \cdot \vec{\partial} \partial_{0}^{2} V+\frac{5}{4}\left(\partial_{0}^{2} V\right)^{2}-\frac{1}{16} \partial_{0}^{6} V\right)+\left\{\begin{array}{c}
5 \\
-7
\end{array}\right\} \frac{3}{4} V\left(\partial_{0} V\right)^{2} \\
\left.+\left\{\begin{array}{c}
7 \\
-11
\end{array}\right\} \frac{5}{64}\left(\vec{\partial} \partial_{0} V\right)^{2}+\left\{\begin{array}{c}
-7 \\
8
\end{array}\right\} \frac{1}{4} \partial_{0} V \partial_{0}^{3} V\right]
\end{aligned}
$$

where the upper coefficients in the braces refer to (space-time) Dirichlet boundary conditions, whereas the lower ones refer to Neumann boundary conditions.

The same results, in the form produced by the DBC propagators, is obtained by adding to the $a_{n}$ suitable vanishing terms, written as total derivatives minus their boundary values. Here we list the first few ones

$$
\begin{aligned}
\Delta a_{2}=0= & -\int_{M} \frac{1}{3 !} \square V-\int_{\partial M} \frac{1}{3 !} \partial_{0} V \\
\Delta a_{5 / 2}=0= & \pm \frac{\sqrt{\pi}}{2} \int_{\partial M} \frac{1}{3 !} \vec{\partial}^{2} V \\
\Delta a_{3}=0= & \int_{M} \frac{1}{3 !} \partial_{\beta}\left(V \partial_{\beta} V-\frac{1}{10} \partial_{\beta} \square V\right)+\int_{\partial M} \frac{1}{3 !}\left(V \partial_{0} V-\frac{1}{10} \partial_{0} \square V\right) \\
& +\int_{\partial M}\left\{\begin{array}{c}
8 \\
-12
\end{array}\right\} \frac{1}{5 !} \partial_{0} \vec{\partial}^{2} V \\
\Delta a_{7 / 2}=0= & \mp \frac{\sqrt{\pi}}{2} \int_{\partial M} \frac{1}{3 !} \partial_{b}\left(V \partial_{b} V-\left(\frac{1}{4} \partial_{0}^{2}+\frac{1}{10} \vec{\partial}^{2}\right) \partial_{b} V\right)
\end{aligned}
$$

that allow to obtain a consistent check with the coefficients computed earlier in [28].

\section{References}

[1] Z. Bern and D.A. Kosower, The computation of loop amplitudes in gauge theories, Nucl. Phys. B 379 (1992) 451.

[2] M.J. Strassler, Field theory without Feynman diagrams: one loop effective actions, Nucl. Phys. B 385 (1992) 145 hep-ph/9205205.

[3] R.D. Ball, Chiral gauge theory, Phys. Rept. 182 (1989) 1.

[4] D.V. Vassilevich, Heat kernel expansion: user's manual, Phys. Rept. 388 (2003) 279 hep-th/0306138.

[5] K. Kirsten, Spectral functions in mathematics and physics, Chapman \& Hall/CRQ, U.S.A. (2001).

[6] C. Schubert, Perturbative quantum field theory in the string-inspired formalism, Phys. Rept. 355 (2001) 73 hep-th/0101036.

[7] D. Fliegner, P. Haberl, M.G. Schmidt and C. Schubert, The higher derivative expansion of the effective action by the string inspired method. II, Ann. Phys. (NY) 264 (1998) 51 hep-th/9707189.

[8] D. Fliegner, M.G. Schmidt and C. Schubert, The higher derivative expansion of the effective action by the string inspired method. Part 1, Z. Physik C 64 (1994) 111 hep-ph/9401221. 
[9] D. Fliegner, P. Haberl, M.G. Schmidt and C. Schubert, An improved heat kernel expansion from worldline path integrals, Discourses Math. Appl. 4 (1995) 87 hep-th/9411177.

[10] M.G. Schmidt and C. Schubert, On the calculation of effective actions by string methods, Phys. Lett. B 318 (1993) 438 hep-th/9309055;

D.G.C. McKeon and T.N. Sherry, Radiative effects in a constant magnetic field using the quantum mechanical path integral, Mod. Phys. Lett. A 9 (1994) 2167;

D. Cangemi, E. D'Hoker and G.V. Dunne, Derivative expansion of the effective action and vacuum instability for QED in (2+1)-dimensions, Phys. Rev. D 51 (1995) 2513 hep-th/9409113;

V.P. Gusynin and I.A. Shovkovy, Derivative expansion for the one-loop effective Lagrangian in QED, Can. J. Phys. 74 (1996) 282 hep-ph/9509383; Derivative expansion of the effective action for QED in $2+1$ and $3+1$ dimensions, J. Math. Phys. 40 (1999) 5406

hep-th/9804143;

R. Shaisultanov, On the string-inspired approach to QED in external field, Phys. Lett. B 378 (1996) 354 hep-th/9512142;

M. Reuter, M.G. Schmidt and C. Schubert, Constant external fields in gauge theory and the spin 0, 1/2, 1 path integrals, Ann. Phys. (NY) 259 (1997) 313 hep-th/9610191.

[11] U. Müller, Basis invariants in non-Abelian gauge theories, DESY-96-154 hep-th/9701124.

[12] F. Bastianelli, The path integral for a particle in curved spaces and Weyl anomalies, Nucl. Phys. B 376 (1992) 113 hep-th/9112035;

F. Bastianelli and P. van Nieuwenhuizen, Trace anomalies from quantum mechanics, Nucl. Phys. B 389 (1993) 53 hep-th/9208059.

[13] F.A. Dilkes and D.G.C. McKeon, Off-diagonal elements of the DeWitt expansion from the quantum mechanical path integral, Phys. Rev. D 53 (1996) 4388 hep-th/9509005;

F. Bastianelli and O. Corradini, On mode regularization of the configuration space path integral in curved space, Phys. Rev. D 60 (1999) 044014 hep-th/9810119; 6D trace anomalies from quantum mechanical path integrals, Phys. Rev. D 63 (2001) 065005 hep-th/0010118;

F. Bastianelli and A. Zirotti, Worldline formalism in a gravitational background, Nucl. Phys. B 642 (2002) 372 hep-th/0205182;

F. Bastianelli, O. Corradini and A. Zirotti, Dimensional regularization for SUSY $\sigma$-models and the worldline formalism, Phys. Rev. D 67 (2003) 104009 hep-th/0211134;

F. Bastianelli, P. Benincasa and S. Giombi, Worldline approach to vector and antisymmetric tensor fields, JHEP 04 (2005) 010 hep-th/0503155; Worldline approach to vector and antisymmetric tensor fields. II, JHEP 10 (2005) 114 hep-th/0510010.

[14] F. Bastianelli, O. Corradini and A. Zirotti, BRST treatment of zero modes for the worldline formalism in curved space, JHEP 01 (2004) 023 hep-th/0312064.

[15] M. Bordag, U. Mohideen and V.M. Mostepanenko, New developments in the Casimir effect, Phys. Rept. 353 (2001) 1 quant-ph/0106045.

[16] M. Fabinger and P. Hořava, Casimir effect between world-branes in heterotic M-theory, Nucl. Phys. B 580 (2000) 243 hep-th/0002073;

J. Garriga, O. Pujolàs and T. Tanaka, Radion effective potential in the brane-world, Nucl. Phys. B 605 (2001) 192 hep-th/0004109;

S. Nojiri, S.D. Odintsov and S. Zerbini, Bulk versus boundary (gravitational Casimir) effects in quantum creation of inflationary brane world universe, Class. and Quant. Grav. 17 (2000) 4855 hep-th/0006115; 
R. Hofmann, P. Kanti and M. Pospelov, (De-)stabilization of an extra dimension due to a Casimir force, Phys. Rev. D 63 (2001) 124020 hep-ph/0012213].

[17] H. Gies and K. Langfeld, Quantum diffusion of magnetic fields in a numerical worldline approach, Nucl. Phys. B 613 (2001) 353 hep-ph/0102185; Loops and loop clouds: a numerical approach to the worldline formalism in QED, Int. J. Mod. Phys. A 17 (2002) 966 hep-ph/0112198.

[18] H. Gies, K. Langfeld and L. Moyaerts, Casimir effect on the worldline, JHEP 06 (2003) 018 hep-th/0303264;

H. Gies and K. Klingmuller, Worldline algorithms for Casimir configurations, Phys. Rev. D 74 (2006) 045002 quant-ph/0605141.

[19] P. Hořava and E. Witten, Heterotic and type-I string dynamics from eleven dimensions, Nucl. Phys. B 460 (1996) 506 hep-th/9510209]; Eleven-dimensional supergravity on a manifold with boundary, Nucl. Phys. B 475 (1996) 94 hep-th/9603142.

[20] L. Álvarez-Gaumé, Supersymmetry and the Atiyah-Singer index theorem, Commun. Math. Phys. 90 (1983) 161;

L. Álvarez-Gaumé and E. Witten, Gravitational anomalies, Nucl. Phys. B 234 (1984) 269

D. Friedan and P. Windey, Supersymmetric derivation of the Atiyah-Singer index and the chiral anomaly, Nucl. Phys. B 235 (1984) 395.

[21] H.P. McKean and I.M. Singer, Curvature and the eigenvalues of the Laplacian, J. Diff. Geom. 1 (1967) 43.

[22] G. Kennedy, R. Critchley and J.S. Dowker, Finite temperature field theory with boundaries: stress tensor and surface action renormalization, Ann. Phys. (NY) 125 (1980) 346.

[23] T.P. Branson and P.B. Gilkey, The asymptotics of the Laplacian on a manifold with boundary, Commun. Part. Diff. Equat. 15 (1990) 245.

[24] G. Cognola, L. Vanzo and S. Zerbini, A new algorithm for asymptotic heat kernel expansion for manifolds with boundary, Phys. Lett. B 241 (1990) 381.

[25] D.M. McAvity and H. Osborn, A DeWitt expansion of the heat kernel for manifolds with a boundary, Class. and Quant. Grav. 8 (1991) 603 [Erratum ibid. 9 (1992) 317]; Asymptotic expansion of the heat kernel for generalized boundary conditions, Class. and Quant. Grav. 8 (1991) 1445;

D.M. McAvity, Heat kernel asymptotics for mixed boundary conditions, Class. and Quant. Grav. 9 (1992) 1983 .

[26] T.P. Branson, P.B. Gilkey and D.V. Vassilevich, The asymptotics of the Laplacian on a manifold with boundary. 2, Boll. Union. Mat. Ital. 11B (1997) 39 hep-th/9504029;;

K. Kirsten, The $A_{5}$ heat kernel coefficient on a manifold with boundary, Class. and Quant. Grav. 15 (1998) L5 hep-th/9708081.

[27] J.S. Dowker and K. Kirsten, Smeared heat-kernel coefficients on the ball and generalized cone, J. Math. Phys. 42 (2001) 434 hep-th/9803094;

T.P. Branson, P.B. Gilkey, K. Kirsten and D.V. Vassilevich, Heat kernel asymptotics with mixed boundary conditions, Nucl. Phys. B 563 (1999) 603 hep-th/9906144.

[28] F. Bastianelli, O. Corradini and P.A.G. Pisani, Worldline approach to quantum field theories on flat manifolds with boundaries, JHEP 02 (2007) 059 hep-th/0612236].

[29] F. Bastianelli, O. Corradini and P.A.G. Pisani, Scalar field with Robin boundary conditions in the worldline formalism, J. Phys. A 41 (2008) 164010 arXiv:0710.4026. 